286 A mmelburg: $\alpha_{1}-\alpha_{2}$-Amidoacetnaphtalidmonosulfos.

\title{
Ueber die $\alpha_{1}-\alpha_{2}$-Amidoacetnaphtalidmonosulfosäure des Patents Nr. 66354; \\ von
}

\section{A. Ammelburg.}

Im Patent Nr. 66354 rom 19. December 1891 beschreiben Dahl u. Co. in Barmen die Darstellung einer Amidoacetnaphtalidmonosulfosäure. Ueber die Stellung der Sulfongruppe war bisher nichts bekannt; die Bestimmung derselben bildet den wesentlichsten Theil der vorliegenden Untersuchung, welche ich auf Anregung des Hrn. Dr. Gaess ausführte.

Nach Angabe der Patentschrift wird das Amidoacetnaphtalid erhalten durch Reduction des $\alpha_{1}-\alpha_{2}$-Nitroacetnaphtalids, und lässt sich durch rauchende Schwefelsäure leicht in eine Sulfosäure überführen.

Man rührt das in Wasser schwer lösliche Sulfat in die 3-4 fache Menge 20\% Anhydrid enthaltender Schwefelsäure, unter Vermeidung einer Temperaturerhöhung über $25^{\circ}-30^{\circ}$, und erwärmt nach der Lösung noch so lange auf $40^{\circ}-50^{\circ}$, bis sich kein Amidoacetnaphtalid mehr nachweisen lässt.

Die Sulfosäure bildet im freien, reinen Zustand ein in Wasser schwer lösliches Krystallpulver; einmal gelöst, bleibt sie jedoch leicht in Lösung und krystallisirt in kleinen, farblosen Nadeln aus. Die Alkalisalze der Säure sind in Wasser sehr leicht löslich; ebenso die Salze der Erden.

Eisenchlorid oder Chromsäurelösung färbt die Lösung der Sulfonsäure prachtroll blau. ${ }^{1}$ ) Zur Vervollständigung der Kenntniss der Säure stellte ich noch einige Salze dar.

Das Barytsalz der Amidoacetnaphtalidmonosulfósäure wurde erhalten, indem ein Theil Säure in Wasser suspendirt und unter Erwärmen mit der berechneten Menge Aetzbaryt nach und nach versetzt wurde. Die Säure löst sich hierbei glatt; alsdann wurde noch kurze Zeit Kohlensäure in die Lösung eingeleitet, um etwaigen im Ueberschuss vorhandenen Aetzbaryt auszufällen. Nach dem Filtriren wurde die klare Lösung mit Kohle behandelt und bis zur beginnenden Krystal-

1) Die chem. Industrie 1893, S. 89 . 
Ammelburg: $a_{1}-a_{2}$-Amidoacetnaphtalidmonosulfos. 287

lisation eingedampft. Bei sehr langsamer Krystallisation erhält man das Barytsalz in schönen, grossen, verwachsenen Tafeln, sonst in farblosen, büschelförmig verwachsenen, mikroskopischen Nädelchen. Das Barytsalz ist in kaltem und heissem Wasser sehr leicht löslich und krystallisirt mit $7 \mathrm{Mol}$. Krystallwasser.

Wasserbestimmung:

Angewandte Substanz, lufttrocken . . . . . . . 0,4693 Grm. Gefunden Krystallwasser nach 6 stündig. Trocknen bei $120^{\circ} \quad 0,0702$ " Berechnet auf 7 Mol. Krystallwasser . . . . . . . 15,33\%. Gefunden

Barytbestimmung:

Angewandte Substanz . . . . . 0,4943 Grm.

Gefunden $\mathrm{BaSO}_{4}$. . . . . . . . 0,1389 "

Dies entspricht . . . . . . . . 0,0816 "Ba.

Gefunden . . . . . . . . 16,5 $\%$ Ba.

Berechnet . . . . . . . . . . 16,5 "Ba.

Das Kalisalz, aus dem Barytsalz durch Umsetzung mit kohlensaurem Kali dargestellt, krystallisirt in farblosen, mikroskopischen Nädelchen, welche kein Krystallwasser enthalten und sehr leicht in Wasser löslich sind.

Kalibestimmung:

Angewandte Substanz . . . . 0,3411 Grm.

Gefunden $\mathrm{K}_{2} \mathrm{SO}_{4}$. . . . . . . 0,0916 "

Dies entspricht . . . . . . . 0,0410 "Kali.

Gefunden . . . . . . . . 12,01\% Kali.

Berechnet . . . . . . . . . 12,2 "Kali.

Das Zinksalz, ebenfalls aus dem Barytsalz mit schwefelsaurem Zink erhalten, ist leicht löslich in Wasser und krystallisirt daraus in glänzenden, farblosen Nadeln mit $3^{1 / 2} \mathrm{Mol}$. Krystallwasser.

Wasserbestimmung:

Angewandte Substanz, lufttrocken . . . . . . . 0,1531 Grm. Gefunden Krystallwasser nach 6stündig. Erhitzen auf $120^{\circ} 0,0141$ " Berechnet auf $3 \frac{1}{2}$ Mol. Krystallwasser . . . . . . 9,18\%. Gefunden . . . . . . . . . . . . . 9,20 ".

Zinkbestimmung:

Angewandte Substanz . . . . 0,1390 Grm.

Gefunden $\mathrm{ZnSO}_{4}$. . . . . . 0,0320 "

Dies entspricht . . . . . . . 0,0129 " Zn

Gefunden . . . . . . . 9,3\% Zn.

Berechnet . . . . . . . $9,47 \% \mathbf{Z n}$. 
288 Ammelburg: $a_{1}-a_{2}$-Amidoacetnaphtalidmonosulfos.

Das Bleisalz, dargestellt aus dem Barytsalz mit salpetersaurem Blei, ist in Wasser bedeutend schwerer löslich, als die anderen Salze und krystallisirt bei längerem Stehen aus seinen Lösungen, selbst aus verdünnten aus. Es krystallisirt aus $W$ asser in kleinen, mikroskopischen Tafeln mit $11 / 2$ Mol. Krystallwasser.

Für die Ableitung der Constitution der Sulfonsäure war es zunächst von Wichtigkeit, festzustellen, ob sich die Sulfongruppe in dem die Amidogruppen enthaltenden, oder in dem anderen Kern des Naphtalins befindet.

Zu dem Zweck wurde zur Oxydation der Amidoacetnaphtalidmonosulfosäure mit Kaliumpermanganat in alkalischer Lösung geschritten.

10 Grm. Säure wurden in Ammoniak gelöst und alsdann nach und nach bei gewöhnlicher Temperatur $685 \mathrm{Ccm}$. einer 4 procent. Kaliumpermanganatlösung zugegeben. Nach Zugabe der $685 \mathrm{Ccm}$. verschwand die Rothfärbung nicht mehr. Es wurde hierauf rom ausgeschiedenen Braunstein abfiltrirt, das Filtrat zur Trockne verdampft, mit Salzsäure angesäuert und mit Aether ausgeschüttelt.

Nach dem Verdunsten des Aethers hinterblieben geringe Mengen einer gelben Krystallmasse, welche sich in Aether sehr schwer, leichter dagegen in Wasser löste. Die hieraus ausgeschiedenen Krystalle zeigten nach dem Sublimiren den Schmelzpunkt von Phtalsäureanhydrid, nämlich $128^{\circ}$.

Beim nächsten Versuch, die Säure mit Kaliumpermanganat in alkalischer Lösung zu oxydiren, wurde, sobald bei gewöhnlicher Temperatur die Rothfärbung nicht mehr verschwand, auf dem Wasserbad erwärmt. Ich brauchte diesmal auf $6 \mathrm{Grm}$. Naphtylendiaminsulfosäure $1005 \mathrm{Ccm}$. 4 procent. Kalipermanganatlösung. Dies entspricht $38 \mathrm{Grm}$. oder 9,6 Mol. $\mathrm{KMnO}_{4}$.

Im Uebrigen wurde genau so verfahren, wie beim vorhergehenden Versuch, und auch diesmal erhielt ich geringe Spuren Phtalsäure rom Schmelzp. 210 , sublimirt bei $128^{\circ}$. Zum genaueren Beweis, dass die erhaltene Säure wirklich Phtalsäure sei, führte ich obendrein die Fluoresceïnreaction aus.

Bei der äusserst geringen Menge Phtalsäure, welche ich 
Ammelburg: $\alpha_{1}-\alpha_{2}$-Amidoacetnaphtalidmonosulfos. 289 erhielt, konnte dieselbe unmöglich als Beweis für die Stellung der Sulfogruppe dienen; ich musste ihr Entstehen entweder der Beimengung geringer Mengen einer isomeren zu der den Hauptbestandtheil ausmachenden Säure, oder einer secundären Reaction zuschreiben, und es war anzunehmen, dass als wesentlichstes Oxydationsprodukt eine Phtalsulfonsäure entstanden sei, dass also die Sulfongruppe nicht in demselben Kern wie die Amidogruppen steht. Ein erneuter Versuch bestätigte diese Annahme.

$29 \mathrm{Grm}$. Amidoacetnaphtalidsulfosäure wurden in der vorbeschriebenen Art oxydirt; den abgeschiedenen Braunstein filtrirte ich $a b$ und dampfte das Filtrat zur Trockne ein. Der Rückstand wurde mit $75 \mathrm{Grm}$. Aetzkali versetzt, und dieses Gemisch in einer Nickelschale bei einer Temperatur von $200^{\circ}-220^{\circ}$ unter fleissigem Umrühren geschmolzen, bis eine herausgenommene Probe beim Ansäuern mit verdünnter Schwefelsäure stark schweflige Säure entwickelte. Alsdann liess ich erkalten, nahm die Schmelze mit heissem Wasser auf und sänerte mit verdünnter Schwefelsäure stark an. Es schied sich hierbei eine gelblich weisse Masse ab, welche anorganisch war. Nachdem durch die überstehende Lösung zur Entfernung der schwefligen Säure Luft gesaugt worden war, wurde durch wiederholtes Ausschütteln mit Aether die darin gelöste organische Substanz ausgezogen. Der nach dem Abdestilliren des Aethers zurückbleibende Rückstand wurde mit Wasser aufgenommen, mit Kohle gekocht, filtrirt, und das farblose Filtrat der fractionirten Krystallisation unterworfen. Die einzelnen Fractionen schmolzen bei langsamem Erhitzen unter Aufschäumen bei $185^{\circ}$.

Die geschmolzen gewesene Substanz zeigt den Schmelzp. $164^{\circ}-165^{\circ}$. In kaltem Wasser löst sie sich schwer, leicht in heissem und krystallisirt aus der heissen wässrigen Lösung beim Erkalten in kurzen, zu Rosetten vereinigten Spiessen aus. Eisenchlorid bewirkt in der wässrigen Lösung eine rothgelbe Färbung. Diese Erscheinungen stimmen genau mit den Angaben über $\beta$-Oxyphtalsäure überein.

Die Analyse bestätigt die Voraussetzung:

Berechnet auf $\mathrm{C}_{9} \mathrm{H}_{6} \mathrm{O}_{\overline{5}}$ ergaben 0,2992 Grm. angewandter Substanz folgendes Resultat. 
290 Ammelburg: $\alpha_{1}-\alpha_{2}$-Amidoacetnaphtalidmonosulfos.

$\begin{array}{ccc} & \text { Berechnet: } & \text { Gefunden: } \\ \text { C } & 52,7 & 52,5 \% \\ \text { H } & 3,29 & 3,5 \%\end{array}$

Die Ausbeute an Säure betrug $10 \mathrm{Grm}$. aus $29 \mathrm{Grm}$. amidoacetnaphtalidsulfonsaurem Baryt.

Ausserdem stellte ich aus dem Silbersalz meiner Säure vermittelst Jodmethyl im Rohr noch den neutralen Dimethylester dar. Nach zweimaligem Umkrystallisiren aus Wasser erhielt ich denselben in farblosen, grossen, glänzenden Tafeln, welche den constanten Schmelzp. 104,50 zeigen. Während Krystallform und die Löslichkeit mit den Angaben von A. Ries ${ }^{1}$ ) übereinstimmen, fand ich den Schmelzpunkt zwei Grad höher.

Nachdem somit festgestellt war, dass die Sulfogruppe bei der $\alpha_{1}-\alpha_{2}$-Amidoacetnaphtalidmonosulfosäure sich in einer $\beta$-Stellung des nicht die Amidogruppen enthaltenden Naphtalinkerns befindet, blieb nur noch zu bestimmen, ob die Sulfogruppe die Stelle 6 oder 7 einnimmt.

Zum Ziele mussten zwei Wege führen:

I. Der Abbau der Amidoacetnaphtalidmonosulfosäure zur Amidonaphtalinsulfosäure und Vergleichung dieser Säure mit den neuerdings von Erdmann genau beschriebenen Naphtylaminmonosulfosäuren. ${ }^{2}$ )

II. Die Umwandlung der erbaltenen Naphtylaminmonosulfosäure in das entsprechende Dichlornaphtalin und Identificirung desselben.

Ich verfuhr folgendermassen:

23 Grm. Barytsalz der Amidoacetnaphtalidmonosulfosäure wurden in etwas Wasser gelöst und hierauf 23 Grm. 40 proc. Salzsäure zugesetzt.

Alsdann wurde in der Kälte diazotirt und auf bekannte Weise vermittelst zweier Moleküle in Salzsäure gelösten Zinnchlorürs die Diazogruppe in die Hydrazingruppe übergeführt.

Die Acetnaphtalidhydrazinsulfosäure ist schwer löslich und fällt nach 12 stiundigem Stehen nahezu quantitativ aus dem Reactionsgemisch aus. Unter der Lupe erscheint sie in der Form farbloser, kleiner Nädelchen, unter dem Mikroskop dagegen in der schmaler, langgestreckter Tafeln.

1) Ann. Chem. 233, $\left.233 . \quad{ }^{2}\right)$ Das. 275, 205 u. 272. 
Ammelburg: $a_{1}-a_{2}$-Amidoacetnaphtalidmonosulfos. 291

$10 \mathrm{Grm}$. Acetnaphtalidhydrazinsulfosäure wurden in einem Liter Wasser, dem 24 Grm. 40procent. Salzsäure zugesetzt waren, suspendirt und zum Sieden erhitzt. Hierauf setzte ich dem heissen Gemisch behufs Eliminirung der Hydrazingruppe so lange frisch bereitete Kupferchloridlösung tropfenweise zu, als noch Stickstoff entwich; dann wurde in der Wärme Schwefelwasserstoff eingeleitet, vom ausgeschiedenen Schwefelkupfer abfiltrirt, und das Filtrat zur Trockne eingedampft.

Ich erhielt aus $10 \mathrm{Grm}$. angewandter Substanz $7 \mathrm{Grm}$. Amidonaphtalinsulfosäure. Ausser der Hydrazingruppe war auch die Acetylgruppe ausgetreten.

Die erhaltene Naphtylaminmonosulfosäure ist in kaltem Wasser ziemlich schwer, in heissem ziemlich leicht löslich und krystallisirt daraus in glänzenden Nädelchen. Die Lösungen der Säure und ihrer Alkalisalze fluoresciren grün.

Eisenchlorid und Goldchlorid bewirken in verdünnten wässrigen Lösungen prächtige Blaufärbungen, welche sich nicht wesentlich von einander unterscheiden. Durch Essigsäure schlägt die mit Eisenchlorid hervorgebrachte Blaufärbung nach einigem Stehen in Roth um.

Die Diazoverbindung der Säure ist in Wasser sehr leicht löslich. Die Säure in verdünnter Salzsäure suspendirt, löst sich bei Zugabe von Natriumnitrit mit intensiv goldgelber Farbe auf.

$\alpha$-Naphtol in alkalischer Lösung giebt mit der Diazosäure einen leuchtend rothen Farbstoff, welcher durch Essigsäure gelb wird.

Diese Eigenschaften der von mir erhaltenen Säure stimmen mit den von Erdmann für 1:7-Naphtylaminsulfosäure ${ }^{1}$ ) angegebenen genau überein; es dürfte somit der Amidoacetnaphtalidmonosulfosäure die Constitution

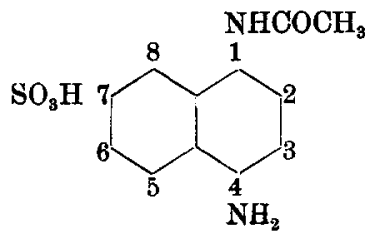

1) Herr Dr. H. Erdmann hatte die Güte, mir eine Probe 1:7Naphtylaminsulfosäure zur Verfügung zu stellen, wofür ich ihm meinen besten Dank ausspreche. 
292 Ammelburg: $a_{1}-a_{2}$-Amidoacetnaphtalidmonosulfos. zuzuschreiben sein, wobei jedoch nicht übersehen werden darf, dass die Naphtylaminsulfosäure 1:7 eine sehr weit gehende Aehnlichkeit mit der 1:6-Säure besitzt, so dass es zum sicheren Stellungsnachweis noch weiterer Beweise bedarf.

Am geeignetsten dazu erschien mir, wie oben bemerkt, die Darstellung des Dichlornaphtalins aus der Naphtylaminmonosulfosäure. Zur Gewinnung desselben wurden $7 \mathrm{Grm}$. Naphtylaminmonosulfosäure diazotirt, und die in Lösung befindliche Diazoverbindung tropfenweise $\mathrm{zu} 60$ Grm. einer 10 procent. heissen Kupferchlorürlösung laufen gelassen. Nach dem Entfernen des Kupfers mit Schwefelwasserstoff wurde eingedampft, und der trockne Rückstand wiederholt mit absolutem Alkohol ausgekocht. Nach dem Abdestilliren des Alkohols erhielt ich eine Chlornaphtalinmonosulfosäure, welche ich in ein Dichlornaphtalin überzuführen versuchte.

$3 \mathrm{Grm}$. Chlornaphtalinsulfosäure wurden in einer Retorte mit etwas Phosphoroxychlorid überschichtet und darauf $10 \mathrm{Grm}$. Phosphorpentachlorid zugefügt. Nachdem die eingetretene Reaction beendet war, wurde noch einige Zeit auf eine Temperatur von $120^{\circ}$ erwärmt, und dann die flüchtigen Phosphorverbindungen abdestillirt. Der zurückbleibende Retorteninhalt. wurde mit Wasserdampf behandelt.

Ich erhielt dabei sehr geringe Mengen gechlorter mit Wasserdampf flüchtiger Produkte, welche eine eingehendere Untersuchung nicht gestatteten.

In Folge Semesterschlusses bin ich gezwungen, meine Arbeit abzubrechen; ich hoffe demnächst die Untersuchung. zu Ende führen zu können.

Freiburg, Ende Juli 1893. 